\title{
Clinical trial data for all drugs in current use
}

\author{
Must be made available for independent scrutiny
}

\author{
Fiona Godlee editor in chief
}

BMJ, London WC1H 9JR, UK

The drug industry does many good things. It produces medicines that can improve health and save lives. It creates jobs and stimulates economic growth. Sadly it does bad things too. Persistently and systematically over decades it has withheld and misreported data from clinical trials. ${ }^{1}$ As a result, a whole range of widely used drugs across all fields of medicine have been represented as safer and more effective than they are, endangering people's lives and wasting public money. Such wilful distortion is scientific misconduct. ${ }^{2}$ It is not something we can forgive because of the good things drug companies do. As Ben Goldacre says in the introduction to his new book Bad Pharma, "Drug companies around the world have produced some of the most amazing innovations of the past fifty years, saving lives on an epic scale. But that does not allow them to hide data, mislead doctors, and harm patients."

Hats off then to GlaxoSmithKline, which announced last month that it would allow access to anonymised patient level data from its clinical trials. ${ }^{4}$ An independent panel will assess all requests, and the company's chief executive officer, Andrew Witty, says access will be granted on the basis of a reasonable scientific question, a protocol, and a commitment from the researchers to publish their results. Trial data collected since 2007 will be placed on a password protected website. Earlier data, not yet available in standard digitised formats, will be made available on "an ad hoc basis."

Whether researchers will find it as easy to get past the panel as Witty suggests we will have to wait and see. It will be particularly important to know how many requests are turned down and for what reasons.

And amid the plaudits, a moment of doubt. Surely what this apparently brave and benevolent action really serves to highlight is the rank absurdity of the current situation. Why aren't all clinical trial data routinely available for independent scrutiny once a regulatory decision has been made? How have commercial companies been allowed to evaluate their own products and then to keep large and unknown amounts of the data secret even from the regulators? Why should it be up to the companies to decide who looks at the data and for what purpose? Why should it take legal action (as in the case of GlaxoSmithKline's paroxetine and rosiglitazone),${ }^{56}$ strong arm tactics by national licensing bodies (Pfizer's reboxetine), ${ }^{7}$ and the exceptional tenacity of individual researchers and investigative journalists (Roche's oseltamivir) ${ }^{8}$ to try to piece together the evidence on individual drugs?

Goldacre's book makes it clear that the reasons are complex and there are no simple solutions. But there is no doubt that medical journals could do more. Rather than no longer publishing industry funded trials, as some have suggested, they could leverage their power and publish only where there is a commitment to make the relevant anonymised patient level data available on reasonable request. The International Committee of Medical Journal Editors has so far declined to take such a step. The $B M J$ will require this commitment for all clinical trials of drugs and devices-whether industry funded or not-from January 2013.

The $B M J$ is also intensifying its efforts to help resolve a three year battle to gain access to the full data on oseltamivir (Tamiflu). In 2009 the Cochrane respiratory group, led by Tom Jefferson, was commissioned by the UK government to update its systematic review of neuraminidase inhibitors. Despite a public promise to release "full study reports" (internal company reports) for each trial, each of which can run to thousands of pages, ${ }^{8}$ Roche has stonewalled, variously pleading patient or commercial confidentiality, or claiming that sufficient data have already been provided. ${ }^{9}$

In fact the Cochrane group has told the $B M J$ that about $60 \%$ of Roche's data from phase III trials of oseltamivir have never been published. And although the European Medicines Agency (EMA) could have requested these data from Roche, it did not do so. This means that tax payers in the United Kingdom and around the world have spent billions of dollars stockpiling a drug for which no one except the manufacturer has seen the complete evidence base. Indeed the EMA's unprecedented infringement proceedings launched against Roche last month suggest that even the manufacturer has never fully evaluated evidence it has collected on the drug's adverse effects. ${ }^{10}$ What has Roche got to hide?

Two weeks ago in an attempt to break the deadlock, the $B M J$ wrote to one of the UK's leading academics, John Bell, regius professor of medicine at Oxford University, who is a member of Roche's board of directors. The letter is published this week. ${ }^{11}$ 
In a response not for publication, Bell said he has referred the matter to Roche and is awaiting a response.

Meanwhile, frustrated by the lack of progress, Jefferson and colleagues have given the $B M J$ their entire email correspondence with Roche, which is now published at bmj.com/tamiflu, as David Payne explains. ${ }^{12}$ They have also shared with us their correspondence with the World Health Organization and US Centers for Disease Control and Prevention. The emails show that none of the Cochrane group's questions have been answered. All future emails to and from the Cochrane group will be added to the site.

The open correspondence on bmj.com aims to hold specific individuals and organisations to account. Their actions are preventing independent scrutiny of the results of clinical trials and putting patients' lives at risk. We also hope it will contribute to a sea change in the public mood. Goldacre's book presents an opportunity to raise awareness of a scandal too long ignored by those in power. We should seize this moment with both hands.

Competing interests: The author has completed the ICMJE uniform disclosure form at www.icmje.org/coi_disclosure.pdf (available on request from the corresponding author) and declares: no support from any organisation for the submitted work; no financial relationships with any organisations that might have an interest in the submitted work in the previous three years; no other relationships or activities that could appear to have influenced the submitted work.

Provenance and peer review: Commissioned; not externally peer reviewed.

1 McGauran N, Wieseler B, Kreis J, Schüler YB, Kölsch H, Kaiser T. Reporting bias in medical research-a narrative review. Trials 2010;11:37.

2 A consensus statement on research misconduct in the UK. BMJ 2012;344:e1111.

3 Goldacre B. Bad Pharma. Fourth Estate, 2012.

4 Coombes R. GlaxoSmithKline grants researchers access to clinical trial data. $B M J$ 2012;345:e6909.

5 Lenzer J. Manufacturer admits increase in suicidal behaviour in patients taking paroxetine. BMJ 2006;332:1175.1.

6 Cohen D. Rosiglitazone: what went wrong? BMJ 2010;341:c4848.

7 Wieseler B, McGauran N, Kaiser T. Finding studies on reboxetine: $a$ tale of hide and seek. BMJ 2010;341:c4942.

8 Doshi P. Neuraminidase inhibitors-the story behind the Cochrane review. BMJ 2009;339:b5164.

9 Doshi P, Jefferson T, Del Mar C. The imperative to share clinical study reports: recommendations from the Tamiflu experience. PLoS Med 2012;9:e1001201.

10 Falconi M. Roche faces probe over safety data. Wall Street Journal 2012 Oct 23. http:// online.wsj.com/article/SB10001424052970203406404578074471363752046.html.

11 Godlee F. Open letter to Roche about oseltamivir trial data. BMJ 2012;345:e7305.

12 Payne D. Tamiflu: the battle for secret drug data. BMJ 2012;345:e7303.

Cite this as: BMJ 2012;345:e7304

๑ BMJ Publishing Group Ltd 2012 